\title{
The Impact of Binge Eating Disorder
}

\author{
Yitong Chen ${ }^{1, a, *, \dagger}$, Yushan Liu ${ }^{2, *}, \dagger$, Yinghao Yang ${ }^{3, b, *, \dagger}$
}

\author{
${ }^{1}$ Shanghai Honor School, Shanghai, China \\ ${ }^{2}$ Beijing Royal School, Beijing, China \\ ${ }^{3}$ The Ohio State University, Columbus, Ohio, The United States of America \\ *Corresponding author. Email: ajennyvip@qq.com, byang.5022@osu.edu
}

Those authors contributed equally.

\begin{abstract}
Binge Eating Disorder (BED) is a newly characterized eating disorder, which one will consume a large amount of food, and stop eating until feeling uncomfortably full. One will feel uncontrolled when eating food. This review is trying to give an overview of Binge Eating Disorder, and sum the finding which had in this field, which can give other researchers ideas about their studying direction. In this review, the diagnose of BED had been introduced, some models which about why one started a binge eating episode are also included. The effects of BED are discussed in many different perspectives, the relationships between BED and depression, influences of BED on social relationship, and one's working function can be impacted by BED. The gender difference in BED is also introduced in this section. In addition, prevention and treatment of BED are introduced. The future study direction is discussed, finding out the cause of BED might be finished in the future.
\end{abstract}

Keywords: Binge Eating Disorder, diagnose, Treatment

\section{INTRODUCTION}

According to World Health Organization, the main population of eating disorders is adolescents and young adults. Among the population which affected by eating disorder, there are more females than males. Conditions such as anorexia nervosa, bulimia nervosa, and binge eating disorder are identified by harmful eating behaviours such as restricting calories or binge eating. An eating disorders are often combined with depression, anxiety and/or substance misuse, which is harmful to health [1].

Eating Disorder (ED) can bring many effects to one's daily life and it is related with many other mental illness. Previous research found ED was significantly associated with a higher likelihood of developing major depression, panic disorder, generalized anxiety disorder, and suicidal [2]. ED often occur during adolescents and young adults, more women will be affect by ED. Student's academic performance can be influence by their concerns of their weight which correlated with disordered eating will be a risk as for university administrators. $17.4 \%$ of women $10.4 \%$ of men reported their concern of their weight affect their academic performance [3].
Binge Eating Disorder (BED) is a newly characterized ED and a subgroup of ED. It is not listed in DSMIIIR and listed in DSM-IV for future studying [3]. This review will give an overview of studies in the field. For people having $\mathrm{BED}$, during a binge eating episode, one will feel uncontrollable when eating and take in more food than most people would eat in the same period and circumstance, and the episode will be at least two days a week for six-month period. [4]

In this review, the diagnose and effects of BED will be introduced, and the prevention and treatment will be summaries. Which can help researchers to find out points they want to study about BED. The effects between BED are discussed from many different perspectives. The relationships between depression and BED has been introduced, and one's social relationship and working function can be affected by BED. The gender differences show up in BED are also included in this part. For the treatment of BED, Cognitive Behavior Therapy (CTB), Interpersonal Psychotherapy, Dialectical Behavioral Psychotherapy, and Group Therapy may give help. As for prevention of BED, creating a support group, physical activity, identify the emotional triggers, and Stress Relaxation and Management Techniques can help to pre- 
vent the start of a binge eating episode, and stop people being largely affected by Binge eating behavior.

\section{THE DIAGNOSE AND EFFECTS OF BINGE EATING DISORDER}

\subsection{Definition and Diagnose of Binge Eating Disorder}

\subsubsection{Definition of Binge Eating Disorder}

Binge eating disorder is defined as severe, lifethreatening, and recurring episodes of eating large amounts of food to the point of discomfort (NEDA, 2021). When people binge eats, they usually feel a loss of control or experience shame, distress, or guilt. This is now one of the most prevalent eating disorders in the United States (NEDA, 2021). Recurrent episodes of eating in a given discrete period of time where the amount of food consumed is greater than what people normally eat within the same time or under the same circumstances are indicative of binge eating disorder. In medical terms, binge eating is characterized by certain conditions, such as eating more quickly than usual, eating until comfortably full, eating a large amount of food until feeling comfortably full, eating a large amount of food even if one is not hungry, or eating due to stress, depression, embarrassment, or feeling guilty or disgusted with oneself. However, it should be noted that binge eating is not related to bulimia nervosa, nor does it occur concurrently with this disorder [5]

\subsubsection{Formation and Diagnose of Binge Eating}

Although binge eating disorder is associated with obesity, weight stigma, and weight cycling, this does not necessarily imply that all obese people suffer from binge eating disorder. Aside from that, about two-thirds of clinically obese people have eating disorders. Binge eating disorder is not limited to being overweight; it can occur at any weight.

Although binge eating is one of the most recently diagnosed mental disorders, there have been studies and observations on the subject. The initial phase of diagnosing a person for a possible binge eating disorder (BED) includes the diagnostic of the individual's sociodemographic profile as well as the development features of binge eating manifestations. The next diagnose stage includes the mechanism approach, in which psychological processes are classified based on the person's emotional reactivity, food craving, food-cue relativity, and cognitive control [6]. According to the model noted in the study of Kober and Boswell (2018), there is an interaction between the person's cognitive control and emotional reactivity, food-cue reactivity, and cravings, which affects the irregularities that trigger binge eating. This is also consistent with the findings of Polivy and Herman
(1993) wherein binge eating is influenced psychologically and there are several models by which this can be presented such as addiction model, conditioning model, affective disorders, regulation model, escape model, dieting model, and biopsychosocial model. The regulation model, for example, suggests that as negative feelings increase, binge eating is triggered, and binge eating function lessens the negative effect of emotions because the attention is focused on eating (distraction), and the comfort brought by food is a person's response mechanism.

One of the studies on binge eating focused on assessing the disorder's prevalence among obese people. Using questionnaires, respondents were given a binge eating scale that evaluates their behavioral manifestations such as amount of food consumed, feelings or cognition during binge episodes, as well as a cognitive factor scale that measures cognitive phenomena related to binge eating such as setting unrealistic dietary standards and low efficacy for sustaining a dietary plan. According to the findings of this study, people who binge eat tend to set unrealistic diet goals, with low efficacy in maintaining these diet plans. The study went on to say that obese people have a higher risk of relapsing on self-control when it comes to eating because of the severity of their binge eating and cognition [8].

\subsection{Effects of Binge Eating Disorder}

\subsubsection{Depression}

According to some studies, interpersonal relationships [7] are actually affected by BED syndrome and other depressed feelings. And there are some studies revealed that there is a strong relationship between patients that are diagnosed with binge eating disorder and depression syndrome. Therefore, researchers found a "reciprocal" relationship between depression and binge eating disorder. Although it is still unclear whether depression triggers binge eating or binge eating leads to depression (Barbara Brody,2014), people start to investigate about the association in a further study (Parent, 2009)

There are several explanations are accepted to realize the relationship between BED and depression. One possibility Brody found out is that people need to find a way to cope with their depression. Some of them may lean into the joys of their favorite foods and eat a huge amount to make themselves happier.[8] However, this may lead to a vicious cycle. If people still feel unhappy after eating, they will eat again. Then, people's brains are going to pair happiness with food, and these bad overeating habits will reinforce the depression because people start to feel unsatisfied with their appearance [9]. This is because eating a lot make people become fatter and heavier, so these people start to feel anxious about their body shape due to the pressure from society. So, in this kind of possibility, people first developed depression, 
and they had the binge eating behavior, which led to the strengthen of depression. (Brody, 2014)

\subsubsection{Social Relationships}

Commonly, if a person is diagnosed as binge eating disorder, the relationships with family member and lover will be more and more nervous. Going to encourage the patients to control themselves seems like a right choice, however, this may push them even further away from their close relationship [10]. This is because many of the binge eating behavior happens covertly and exists in the form of isolation for a very long time and people don't need to worry about the types and amount of food they eat. (Zdrodowski (1996) In this way, they will start to avoid all the contact with other people and skirt social activities, and some of them may even lose touch of their families and parents. The negative feeling forced people to do this kind of binge eating, whereas it is the most harmful way to the close relationship and affiliation.

\subsubsection{Work Productivity}

By sending out the international survey by NHWS, 69772 respondents are going to fill out the survey. In BED respondents, the results were showed and compared: there are fewer BED patients than non-BED patients receive the credential for college graduation (Striegel, Bedrosian, Wang 2012), but the percentage of the employment rate doesn't vary so significantly. The income doesn't have large difference, but there is a lack of evidence on the BED sufferers' income information.

Although there is no big gap between the employment rate, the work productivity and absenteeism have a great difference between BED sufferers and non-BED sufferers. The rate of work loss and absenteeism are much higher among the sufferers because of their physical health. And the result also reveals that their daily activities suffered a lot [11].

\subsection{Gender Difference in Binge Eating Disor- der}

According to the nowadays statistics done on the binge eating, women are more likely to report the loss of control when having the behavior of binge eating, while men report overeating more. It is found out that men have minority in reporting the BED. Women are more likely to report the weight dissatisfaction[12], the diet for weight control and the using of purge and the men are likely to control their weights through extreme excess of exercising. Thus, there should be a discussion and experiment done to observe the number of differences in reporting BED between men and women. By comparing to the previous studies, it is found out that there is a significant difference in statistics (overeating once a week:women $10 \%$, men $8 \%$; overeating twice a week: women $7.8 \%$,men $5.8 \%$ ), but the actual behaviours between gender differences are quite little [13].

Causes for gender difference in binge eating can be explain in different perspective. There are some explanations from the biological perspective. One model called developmental mode suggests that the major effect for gender differences in binge eating is because of the testosterone in men and progesterone and estrogen in women (Breedlove 1994, Collaer \& Hines 1995). focus on the psychosocial factor to explain why women are more engaged in the binge eating disorder. These are contained by human as long as they are in the prenatal stage and during puberty, shows the further differentiation. Meanwhile, for female, the risks of developing BED increase when the ovarian hormone activate CNS organizational changes. However, since the testosterone circulate in body and serve as a protection for BED, men are less likely to develop BED in the future when they grow from young kids to young adult [14].

\section{TREATMENT AND PREVENTION FOR BINGE EATING}

\subsection{Treatments for Binge-Eating Disorder}

\subsubsection{Cognitive Behavior Therapy}

Cognitive Behavior Therapy or CBT is a method of psychological treatment that is based on several psychological principles to resolve a wide range of behavioral problems. These principles include psychological problems being based on faulty cognitive behavior or conjecture, psychological problems being based on acquired patterns of behavior, and psychological problems being relieved or resolved through coping mechanisms, which reduces or eliminates the symptoms. The efficacy and effectiveness of cognitive behavior therapy have been shown by research to be comparable to medicinal treatments and other psychological interventions. CBT mainly involves the changing of one's thinking pattern and the modification of one's distorted behavior through various strategies (Palavras et al., 2017) [22]. Based on the findings of Hilbert et al. (2019) [23], cognitive behavioral therapy has been used to treat binge-eating disorder or $\mathrm{BED}$ to great success. CBT addresses and minimizes the dysfunctional behaviors and patterns brought by BED. CBT instills better coping mechanisms concerning stress and depression. CBT prevents distorted mindsets and allows for more effective control over one's actions, including the regulation of one's eating habits. CBT is a strategy utilized by therapists in the real-world setting to address the problems and complications brought upon by BED. 


\subsubsection{Interpersonal Psychotherapy}

Interpersonal psychotherapy is another form of treatment used for a variety of psychosocial problems. It mainly focuses on the improvement of one's relationship with other people, especially friends and family. The primary goal of interpersonal psychotherapy is to create better social relationships and improve interpersonal skills and activity. This reduces anxiety, depression, and other negative psychological states. It also prevents the triggering of binge-eating behavior through the prevention of social anxiety and troubles because of problematic interpersonal relationships and unhealthy communicative patterns (Shafran, 2010) [24]. Interpersonal psychotherapy is being administered by psychotherapists if it fits the situation. Though the long-term effectiveness of the treatment has been questioned, under the right circumstances and methodology, Palvaras et al. (2017) [25] has stated that interpersonal psychotherapy has been shown to greatly improve the behavior, eating patterns, and psychological state of those who suffer from bingeeating disorder.

\subsubsection{Dialectical Behavioral Psychotherapy}

Dialectical Behavioral Therapy or DBT is a derivation of cognitive behavior therapy that strives to allow people to cope with the situation and stress as well as regulate emotions and "live in the moment." Dialectical behavioral therapy has also been shown to improve social relationships. Though dialectical behavioral therapy was initially utilized to treat borderline personality disorder of BPD, its effectiveness branches towards other mental health problems such as binge-eating disorder. It may be used to treat people suffering from selfdestructing behavior, unproductive mindsets, and improper regulation of emotions. Dialectical behavioral therapy is an evidence-based approach that employs various techniques and strategies (Lammers et al., 2020) [26]. The main focuses of dialectical behavioral therapy are mindfulness skills, stress tolerance, and emotion regulation. Mindfulness develops one's ability to perceive the moment and surroundings, which allows a person to slow down, remain calm, assess the situation, and prevent negative patterns such as binge-eating in BED. Stress tolerance teaches preparation and healthy coping techniques in times of emotional distress. This prevents relapsing into binge-eating behavior if one suffers from binge-eating disorder. Lastly, during intense emotional feedback, binge-eating behavior may be triggered. Emotional regulation allows the effective handling and processing of intense emotions that may excite binge-eating disorder (Carter et al. 2020) [27]. Dialectical behavioral therapy is effective in handling the symptoms and treating behavioral problems, such as BED, in real-world situations as shown in several studies, researches, and applicative efforts by professionals (Yacoub, 2019) [28].

\subsubsection{Group Therapy}

Group-based psychological treatments or therapy is an approach in which a therapist handles several people, usually of the same conditions or behavioral problems. Group therapy is very effective in improving mental health by allowing people of similar conditions to work towards particular goals together. Many mental health professionals, according to Tasca et al. (2006) [29], have advocated and utilized group therapy to improve the conditions and psychosocial behavior of people suffering from binge-eating disorder or BED. It elicits a sense of familiarity and community amongst its participants that helps people control their urges and binge-eating behavior. The participants also develop goals and milestones together that further urges them to act, especially when they have others doing the same. Group therapy offers a variety of techniques, activities, strategies that encourage proper control and social responsibility. More importantly, group therapies remove the stigma associated with the condition, which often leads to further deterioration. Based on the study of Boulet (2017) [30], group therapies allow people with the binge-eating disorder to intimately discuss their problems, thoughts, struggles, experiences, and many more. It allows them to vent out their emotional and psychological "backlog." This reduces stress, anxiety, and other behavioral dysfunction, which worsens the condition. This method also creates a safe space with people that understand. Group therapy primarily creates a support structure for people with eating disorders.

\subsubsection{Lisdexamfetamine Dimesylate or Vyvanse}

Vyvanse is an FDA-approved drug that was primarily administered to people with ADHD or attention deficit hyperactivity disorder. According to Heo and Duggan (2017) [31], Lisdexamfetamine dimesylate is a stimulant drug that is used to treat moderate to severe cases of binge-eating disorders amongst adults. It was found to have been well-tolerated in clinical trials on adults and has been shown to effectively treat behaviors regarding binge-eating disorder. However, it is a substance that can be abused and has not been tested on younger patients. Common side-effects include insomnia and dryness of the mouth. However, other serious afflictions have been noted to come from the usage of the drug.

\subsubsection{Topiramate or Topamax}

While not directly used to treat binge-eating disorders, Topiramate or Topamax has been used to alleviate bingeeating episodes as researched by Nourredine et al. (2020) [22]. Primarily an anti-seizure drug, clinical trials and direct administration to patients has been shown to help control binge-eating urges and prevent episodes. However, the safety of the drug has not been fully tested or not- 
ed. Common side-effects include drowsiness, inability to focus, and unstable mood.

\subsubsection{Antidepressants}

Antidepressants are general medications given to those who suffer from depression and its symptoms. According to Stefano et al (2008) [32], the exact mechanisms of how antidepressants work in people with bingeeating disorders are still vague and unknown. Additionally, it has only limited effectiveness when it comes to treating or controlling binge-eating behavior. It is speculated that the mood regulation effects of the drugs are what help sufferers of binge-eating disorders.

\subsection{Preventive Measures for Binge-Eating Dis- order}

\subsubsection{Creation of Support System}

To prevent the development and relapsing of bingeeating disorder or binge-eating behavior, the creation of a social support system is advisable. A support system implies a group of people that understand the condition and are willing to help or provide support in any manner to a person that suffers from the condition. As Compare et al. (2013) [33] has elaborated, many behavioral or mental disorders are alleviated in the presence of a strong support system. It is essential the social connections and relationships that a person forms or has that they can go to for emotional, physical, or practical support. A network of people that helps the person in need or suffering. Highly developed support systems prevent emotional distress, anxiety, problematic thinking, and other dysfunctional patterns that may lead to binge-eating disorder. Not only that but support systems have also been shown to prevent further physical and psychological damage if the person is already suffering from binge-eating disorder. A supportive network of people, including friends and family, allows for an improved mental state, proper venting of distress, realization of various problematic behaviors and situations, correct and objective assessment of the person's state, and support in the various endeavors required for treatment. Lastly, a support system allows the prevention of complications through various methods (Goodrick et al., 1999) [34].

\subsubsection{Active Lifestyle}

According to the studies of Levine et al. (1996) [35], consistent physical activity has been shown to reduce binge-eating behavior and increase control over the binge-eating episodes. Physical activity such as yoga, gym exercise, and running might allow for preventive measures against the development of binge-eating behavior or allow for the prevention of binge-eating episodes. The exact mechanisms are unknown for this phenomenon, but many therapists and mental health professionals have been incorporating physical activities into the practices of treating BED. The possible causes for its preventive effect might $\mathrm{Be}$ due to the release of various hormones and chemicals that help mood regulation and bring a state of euphoria. It might also be possible that the improved body image resulting from physical activity could contribute to the prevention of binge-eating disorder. It is also a recommended approach to reduce bingeeating complications.

\subsubsection{Identification of Emotional Triggers}

Based on the studies of Telch and Agras (1996) [36], some emotional states or triggers result in the provocation of binge-eating behaviors. Triggers that result in emotional distress and anxiety could make people develop binge-eating habits or could cause binge-eating behavior to arise. It is important to determine the possible triggers of stress, provocation, and anxiety to prevent binge-eating episodes. Mental health professionals often assess or help people figure out triggers for sufferers of mental health problems to lessen provocation, to promote techniques to reduce the stress induced by the triggers, and show various methods to avoid the triggers. Identifying the triggers could lessen their effect or could allow people to avoid them so as to not induce a binge-eating episode.

\subsubsection{Stress Relaxation and Management Tech- niques}

Stress-induced binge-eating behavior is one of the most common exhibitions of binge-eating disorder. Stress may trigger mood changes, hormonal or chemical imbalance, and trauma that may push a person to indulge in binge-eating urges. It is very common for people to binge eat because of stress. However, according to Gluck (2006) [37], if stress is managed properly and potentially outputted to more productive or healthy means, bingeeating behavior and complications are prevented. Various techniques that allow people to control, suppress, or manage stress have been discovered and utilized. Some professionals have recommended channeling energy induced by stress into other healthier means.

\section{CONCLUSION}

In this review, the population which have Binge eating disorder had been discussed.Although BED is associated with obesity but this doesn't mean all people who have BED are obsessed, it can occur at any weight.

One can be affected by BED from many perspectives. One may use eating the food they like to stop being sad, this may cause a bad eating habit to form and exacerbate depression. One's relation with family and friends can be influenced by BED. They won't need to think about how much food they will eat when not having a social relationship, thus decreasing their willingness to join in so- 
cial activities. By comparing with women obese without BED, and normal-weight controls, the women who had BED shown a lack of confidence and difficulties in selfexposure to strangers. In addition, a person with BED experiences significant harm in functioning and work productivity when compared with a person without BED. Gender differences are shown in the population of people who have BED. Women are more likely to report uncontrolled eating behavior than men. The gender difference in BED might cause by the physical differences between men and women.

For treating BED, CBT can help to give control to one's action and control binge eating behaviors. Interpersonal psychotherapy can reduce anxiety from social relationships thus control binge eating behaviors. DBT will regulate emotion. Part of the motivation for binge eating behavior is escaping from self-awareness. The common strategy for this is to focus on the present and immediate stimulus environment [39]. DBT may help to make one's social relationship better. DBT may help to stop the relapse of BED. Group therapy let people with a similar condition move in similar direction, and provide support for people who have BED. For treating BED, some drugs can be used.

The prevention for BED may be creating a support system, active lifestyle, identify emotional triggers and techniques that help to release stress. A support system will prevent dysfunctional patterns to cause BED, and protect one from being physical and psychological damage by the disorder. Having an active lifestyle can help one to increase their control of their binge eating behaviour. Identify the triggers like stress can avoid them, and stop the starting of binge eating behaviors. Managing stress can prevent the developed of BED.

BED is a newly characterized eating disorder. Although it can be diagnosed by the, but the cause of BED is still unclear, there are some model being built to find the reason of the stating of a binge eating episode. If BED trigger depression or depression trigger BED is also unclear now, these may be found in the future.

\section{REFERENCES}

[1] https://www.who.int/news-room/factsheets/detail/adolescent-mental-health

[2] Bulik, C. M., Sullivan, P. F., \& Kendler, K. S. (2002). Medical and psychiatric morbidity in obese women with and without binge eating. International Journal of Eating Disorders, 32(1), 72-78. https://doi.org/10.1002/eat.10072

[3] Hoerr, Sharon L.; Bokram, Ronda; Lugo, Brenda; Bivins, Tanya; Keast, Debra R. (2002). Risk for Disordered Eating Relates to both Gender and Ethnicity for College Students. Journal of the American
College of Nutrition, 21(4), 307-314. doi:10.1080/07315724.2002.10719228

[4] Dr. Susan ZelitchYanovski (1993). Binge Eating Disorder: Current Knowledge and Future Directions, 1(4), -. doi:10.1002/j.1550-8528.1993.tb00626.x

[5] Pawaskar, M., Witt, E. A., Supina, D., Herman, B. K., \& Wadden, T. A. (2017). Impact of binge eating disorder on functional impairment and work productivity in an adult community sample in the United States. International Journal of Clinical Practice,

[6] Kober, H., \& Boswell, R. (2018). Potential psychological \& neural mechanisms in binge eating disorder: Implications for treatment. Clinical Psychology Review, 32-44.

[7] Poliy, J., \& P, H. C. (1993). Etiology of binge eating: Psychological mechanisms. In C. G. Fairburn, \& G. T. Wilson, Binge eating: Nature, assessment and treatment (pp. 173-205). Guilford Press.

[8] Gormally, J., Black, S., Daston, S., \& Rardin, D. (1982). The assessment of binge eating severity among obese persons. Addictive Behaviors, 47-55.

[9] Ivanova, Iryna V.; Tasca, Giorgio A.; Hammond, Nicole; Balfour, Louise; Ritchie, Kerri; Koszycki, Diana; Bissada, Hany (2015). Negative Affect Mediates the Relationship Between Interpersonal Problems and Binge-Eating Disorder Symptoms and Psychopathology in a Clinical Sample: A Test of the Interpersonal Model. European Eating Disorders Review, 23(2), 133-138. doi:10.1002/erv.2344

[10] Shapira, N. A., Goldsmith, T. D., \& McElroy, S. L. (2000). Treatment of Binge-Eating Disorder with Topiramate: A Clinical Case Series. The Journal of Clinical Psychiatry, 368-372.

[11] Eisenberg, Daniel; Nicklett, Emily J.; Roeder, Kathryn; Kirz, Nina E. (2011). Eating Disorder Symptoms Among College Students: Prevalence, Persistence, Correlates, and Treatment-Seeking. Journal of American College Health, 59(8), 700 707. doi:10.1080/07448481.2010.546461

[12] De Castro, J. M., Brewer, E. M., Elmore, D. K., \& Orozco, S. (1990). Social facilitation of the spontaneous meal size of humans occurs regardless of time, place, alcohol or snacks. Appetite, 15(2), 89-101. https://doi.org/10.1016/0195-6663(90)90042-7

[13] Pliner, P., \& Bell, R. (2009). A table for one: The pain and pleasure of eating alone. In Meals in Science and Practice (pp. 169-189). Elsevier.

[14] Jacquelyn Ekern, MS, LPC on February 25, 2017

Published on EatingDisorderHope.com, Online 
Information for Eating Disorders

[15] Eating out: The experience of eating in public for the "overweight" woman. (n.d.). Women's Studies International Forum, 19(6), 655-664. https://doi.org/10.1016/S0277-5395(96)00086-6

[16] Pawaskar, Manjiri; Witt, Edward A.; Supina, Dylan; Herman, Barry K.; Wadden, Thomas A. (2017). Impact of binge eating disorder on functional impairment and work productivity in an adult community sample in the United States. International Journal of Clinical Practice, 71(7), e12970-. doi:10.1111/ijcp.12970

[17] Collaer, M. L., \& Hines, M. (1995). Human behavioral sex differences: A role for gonadal hormones during early development? Psychological Bulletin, 118(1), 55-107. https://doi.org/10.1037/00332909.118.1.55

[18] Gormally, J., Black, S., Daston, S., \& Rardin, D. (1982). The assessment of binge eating severity among obese persons. Addictive Behaviors, 47-55.

[19] Ruth H. Striegel-Moore; Francine Rosselli; Nancy Perrin; Lynn DeBar; G. Terence Wilson; Alexis May; Helena C. Kraemer (2009). Gender difference in the prevalence of eating disorder symptoms. , 42(5), 471-474. doi:10.1002/eat.20625

[20] Klump, Kelly L.; Culbert, Kristen M.; Sisk, Cheryl L. (2017). Sex Differences in Binge Eating: Gonadal Hormone Effects Across Development. Annual Review of Clinical Psychology, 13(1), annurevclinpsy-032816-045309-. clinpsy-032816-045309

[21] Nourredine, M., Jurek, L., Auffret, M., Iceta, S., Grenet, G., Kassai, B., Cucherat, M., \& Rolland, B. (2020). Efficacy and safety of topiramate in binge eating disorder: a systematic review and metaanalysis. CNS Spectrums, 1-9.

[22] Hilbert, A., Herpertz, S., Zipfel, S., Tuschen-Caffier, B., Friederich, H.-C., Mayr, A., Crosby, R.D., \& de Zwaan, M. (2019). Early change trajectories in cognitive-behavioral therapy for binge-eating disorder. Behavior Therapy, 50(1), 115-125. [24] Carter, J. C., Kenny, T. E., Singleton, C., Van Wijk, M., \& Heath, O. (2020). Dialectical behavior therapy selfhelp for binge-eating disorder: A randomized controlled study. The International Journal of Eating Disorders, 53(3), 451-460.

[23] Shafran, R. (2010). Interpersonal therapy or guided self-help CBT improve remission from binge eating compared to a behavioural weight-loss programme at 2-year post-treatment. Evidence-Based Mental Health, 13(2), 48.
[24] Palavras, M. A., Hay, P., Filho, C. A. D. S., \& Claudino, A. (2017). The efficacy of psychological therapies in reducing weight and binge eating in people with Bulimia Nervosa and Binge Eating Disorder who are overweight or obese-A critical synthesis and meta-analyses. Nutrients, 9(3), 299.

[25] Lammers, M. W., Vroling, M. S., Crosby, R. D., \& van Strien, T. (2020). Dialectical behavior therapy adapted for binge eating compared to cognitive behavior therapy in obese adults with binge eating disorder: a controlled study. Journal of Eating Disorders, 8(1), 27.

[26] Carter, J. C., Kenny, T. E., Singleton, C., Van Wijk, M., \& Heath, O. (2020). Dialectical behavior therapy self-help for binge-eating disorder: A randomized controlled study. The International Journal of Eating Disorders, 53(3), 451-460.

[27] Yacoub, M. (2019). Adapting dialectical behavior therapy for patients with binge eating and bulimia nervosa disorders. SSRN Electronic Journal. doi:10.2139/ssrn.3457458

[28] Tasca, G. A., Balfour, L., Ritchie, K., \& Bissada, H. (2006). Developmental changes in group The climate in two types of group therapy for binge-eating disorder: A growth curve analysis. Psychotherapy Research: Journal of the Society for Psychotherapy Research, 16(4), 499-514.

[39] Boulet, F. (2017). A cognitive and behavioural group therapy for binge eating disorder: An original 12 sessions design. European Psychiatry: The Journal of the Association of European Psychiatrists, 41(S1), S547-S547.

[30] Heo, Y.-A., \& Duggan, S. T. (2017). Lisdexamfetamine: A review in binge eating disorder. CNS Drugs, 31(11), 1015-1022.

[31] Stefano, S. C., Bacaltchuk, J., Blay, S. L., \& Appolinário, J. C. (2008). Antidepressants in shortterm treatment of binge eating disorder: systematic review and meta-analysis. Eating Behaviors, 9(2), 129-136.

[32] Compare, A., Calugi, S., Marchesini, G., Shonin, E., Grossi, E., Molinari, E., \& Dalle Grave, R. (2013). Emotionally focused group therapy and dietary counseling in binge eating disorder. Effect on eating disorder psychopathology and quality of life. Appetite, 71, 361-368.

[33] Goodrick, G. K., Pendleton, V. R., Kimball, K. T., Carlos Poston, W. S., Reeves, R. S., \& Foreyt, J. P. (1999). Binge eating severity, self-concept, dieting self-efficacy and social support during treatment of 
binge eating disorder. The International Journal of Eating Disorders, 26(3), 295-300.

[34] Levine, M. D., Marcus, M. D., \& Moulton, P. (1996). Exercise in the treatment of binge eating disorder. The International Journal of Eating Disorders, 19(2), 171-177.

[35] Telch, C. F., \& Agras, W. S. (1996). Do emotional states influence binge eating in the obese? The International Journal of Eating Disorders, 20(3), 271279.

[36] Gluck, M. E. (2006). Stress response and binge eating disorder. Appetite, 46(1), 26-30.

[37] Duchesne, M.; de Oliveira Falcone, E. M.; de Freitas, S. R.; D'Augustin, J. F.; Marinho, V.; Appolinario, J. C. (2012). Assessment of interpersonal skills in obese women with binge eating disorder. Journal of Health Psychology, 17(7), 1065-1075. doi:10.1177/1359105311432326 